\title{
Aproximación al manejo de la hiperhidrosis en el pie
}

\author{
An approach to the management of hyperhidrosis in the foot
}

\author{
Daniel López López', Patricia Palomo López ${ }^{2}$, Ángel Morales Ponce ${ }^{3}$, \\ Ruben SÁNCHEZ GóMEZ ${ }^{4}$
}

\author{
${ }^{1}$ Doctor por la Universidade da Coruña. Profesor Asociado. Departamento de Ciencias da Saúde. \\ Facultad de Enfermería e Podoloxía. Universidade da Coruña. daniellopez@udc.es \\ ${ }^{2}$ Doctor por la Universidad Rey Juan Carlos. Profesora Colaboradora. Departamento de Enfermería. \\ Centro Universitario de Plasencia. Universidad de Extremadura. patibiom@unex.es \\ ${ }^{3}$ Doctor por la Universidad Rey Juan Carlos. Clínica Podológica Torrijos, Toledo, España. \\ clinicatorrijos@gmail.com \\ ${ }^{4}$ Doctor por la Universidad Rey Juan Carlos. Profesor Universidad Europea de Madrid. \\ rusago_@hotmail.com
}

Correspondencia:

Dr Daniel López López

Universidade da Coruña

Departamento de Ciencias da Saúde.

Facultad de Enfermaría e Podoloxía.

Campus Universitario de Esteiro s/n

E-15403 Ferrol

Correo electrónico: daniellopez@udc.es

Fecha de recepción: 10 de enero de 2013

Fecha de aceptación: 21 de marzo de 2013

Los autores declaran no tener ningún tipo de interés económico o comercial.

\section{RESUMEN}

La hiperhidrosis es un trastorno patológico causado por un aumento de sudoración superior a las necesidades fisiológicas precisas en el proceso de termorregulación corporal y al balance electrolítico, ya sea de manera local, regional o general. La excesiva sudoración, suele ser bilateral y simétrica relativamente frecuente a nivel general en las axilas, las palmas de las manos, en la región craneofacial y de forma especial en las plantas de los pies. Esta afección ocasiona perturbación ocupacional, deterioro psicológico y físico, y una potencial estigmatización social.

La carencia de consenso en relación a la etiología multifactorial que presenta esta afección y la prevalencia de esta enfermedad a nivel del pie, sobre todo en los jóvenes, nos lleva a mejorar los conocimientos existentes en lo que se refiere a su clasificación, diagnóstico y tratamiento en la búsqueda de actualizar los conceptos relacionados con esta patología del pie.

Palabras clave: sudor; hiperhidrosis; bromohidrosis; hiperhidrosis focal.

\begin{abstract}
Hyperhidrosis is a pathological condition caused by increased sweating than precise physiological needs in the process of body thermoregulation and electrolyte balance, either on a local, regional or general. Excessive sweating is usually bilateral and symmetrical relatively common across the board in the armpits, palms of the hands, in the craniofacial region and especially on the soles of the feet. This condition causes disturbance occupational psychological and physical deterioration, and potential social stigmatization.

The lack of consensus regarding the multifactorial etiology has this condition and the prevalence of this disease at the foot, especially in young people, leads to improved knowledge in regard to their classification, diagnosis and treatment in seeking to update the concepts related to this foot disorders.
\end{abstract}

Key words: sweating; hyperhidrosis; bromohidrosis; focal hyperhidrosis.

Sumario: 1. Introducción. 2. Fisiopatología. 3. Clínica. 4. Diagnóstico. 5. Tratamiento. 6. Conclusiones. Bibliografía.

Referencia normalizada: López López, D., Palomo López, P., Morales Ponce, Á., Sánchez Gómez, R. Aproximación al manejo de la hiperhidrosis en el pie. Rev. Int. Cienc. Podol. 2013; 7(2): 89-97. 


\section{INTRODUCCIÓN}

La hiperhidrosis se define como el aumento de sudoración superior a las necesidades fisiológicas precisas en el proceso de termorregulación corporal y al balance electrolítico, ya sea de manera local, regional o general ${ }^{1,2}$.

La cantidad de secreción de sudor en la superficie cutánea producido ante un estímulo es variable y se establece la presencia de hiperhidrosis cuando el sudor es notorio clínicamente en condiciones en las que normalmente no sería esperable o cuando hay una respuesta desproporcionada a estímulos térmicos, farmacológicos o emocionales ${ }^{3}$.

Además se pueden distinguir una hiperhidrosis primaria, que normalmente es idiopática, caracterizada por un inicio en cualquier momento de la vida, sin causa evidente y localizada a nivel general en las axilas, las palmas de las manos, en el área de la ingle, en la región craneofacial, en el área de los senos y de forma especial en las plantas de los pies, ya sea de forma única en una región o combinada con otras áreas corporales (Figura 1).

También se establece una segunda hiperhidrosis denominada secundaria, que responde a un trastorno subyacente de tipo endocrino, infeccioso o neurológico, generado por diversas causas como la obesidad, el hipertiroidismo,

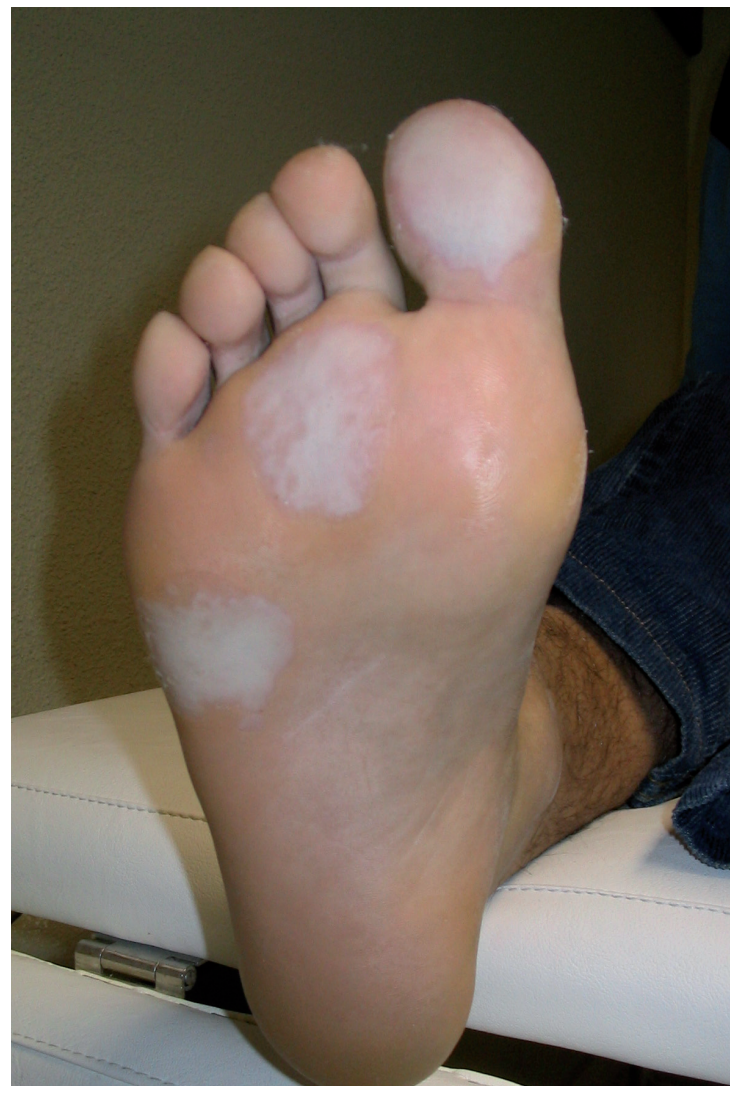

Figura 2. Hiperhidrosis plantar con afectación de queratólisis punctata caracterizada por mal olor.

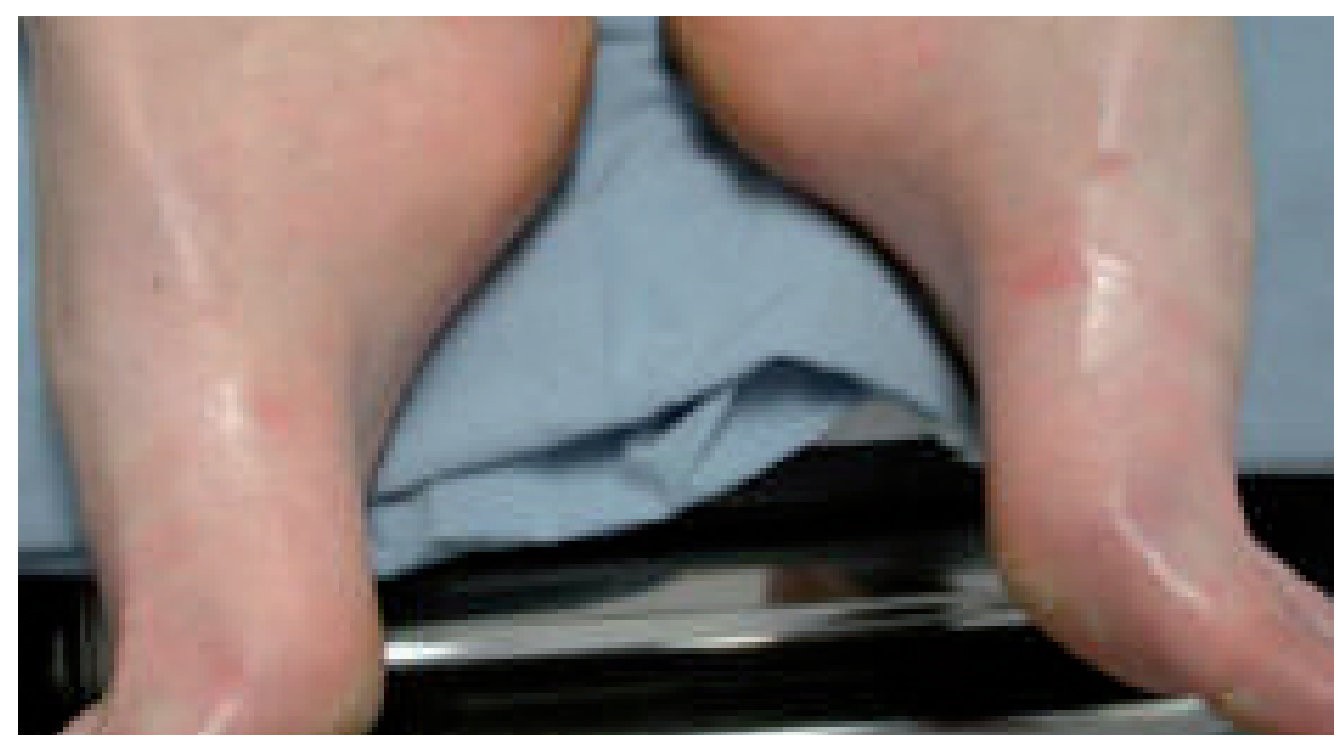

Figura 1. Clínica de la hiperhirdrosis primaria en los pies. 
la diabetes, las alteraciones hormonales como la menopausia, los linfomas, el feocromocitoma, o anormalidades del SNC, etc. Que suele abarcar a todo el cuerpo, comienza en la edad adulta y ocurre tanto durante el sueño como en el estado de vigilia ${ }^{4,5}$.

La prevalencia mundial de la hiperhidrosis es elevada ya que afecta a casi un $3 \%$ de la población general, siendo un motivo principal de consulta debido a que las personas que la padecen se sienten molestas, al interferir su enfermedad en las actividades sociales, laborales y cotidianas, que les puede crear trastornos a nivel cognitivo relacionados con la falta de confianza, los estados de ansiedad y la depresión y a nivel físico relacionado con la maceración y el mal olor de la piel a causa de la humedad persistente de esta, que predispone a la aparición de las infecciones fúngicas o bacterianas de las áreas afectadas conocidas como bromohidrosis (Figura 2) ) $^{6}$.

Se ha constatado una predisposición étnica o genética de la hiperhidrosis auque sigue existiendo una gran controversia debida principalmente a las escasas definiciones de los términos que se utilizan tanto para el diagnóstico del problema y para los tratamientos siendo nuestro propósito realizar una aproximación al manejo de la hiperhidrosis y la repercusión de la enfermedad en el pie pi, $^{8,9}$ que viene apoyada por la necesidad de guías basadas en la evidencia para el tratamiento ${ }^{10}$.

\section{FISIOPATOLOGÍA}

Las glándulas ecrinas les corresponde la misión de producir sudor en el cuerpo, aunque algunos investigadores consideran que esta necesidad es debida a la combinación de ambas (apo / ecrino) que pueden jugar un papel en la hiperhidrosis plantar. A nivel general están distribuidas por toda la superficie corporal, pero en más cantidad en las palmas de las manos, la región craneofacial, el área axilar y en particular en las plantas de los pies. La activación de las mismas, se produce en la etapa de la pubertad siendo las glándulas ecrinas las facilitadoras de la hiperhidrosis focal las cuales se encuentran inervadas por el sistema nervioso simpático, siendo el principal neurotransmisor la acetilcolina. La sudoración térmica se controla por el hipotálamo, mientras que la sudoración emocional está regulada por la corteza cerebral (véase Figura 3) 3,9,11.

La causa de la hiperhidrosis primaria se desconoce, pero parece haber una respuesta hipersensible a los estímulos hipotalámicos producidos y localizados en el córtex cerebral debido a la alta especificidad que suelen condicionar el carácter temprano en la respuesta sudomotora periférica en la hiperhidrosis focal, siendo el análisis genético lo que sugiere que existe un alelo presente en el 5\% de la población afectada de hiperhidrosis y que el $25 \%$ de las personas con una o dos copias del alelo tendrán hiperhidrosis, mientras que menos del $1 \%$ de los personas con dos alelos normales lo tendrán ${ }^{11,12,13}$.

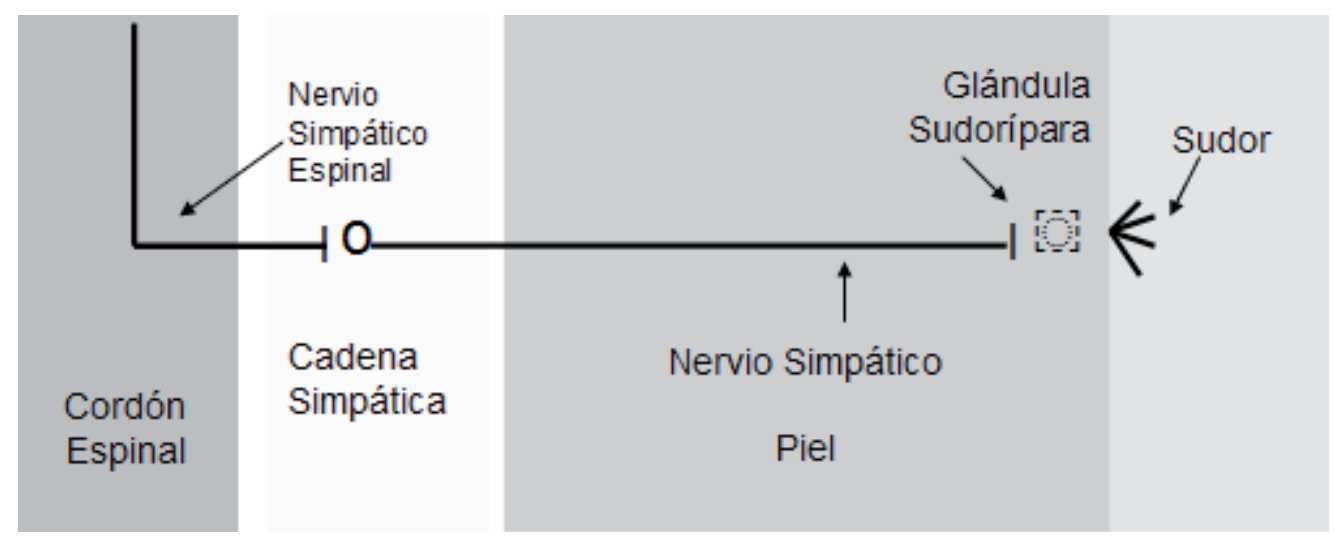

Figura 3. Mecanismo de producción del sudor (De Callejas-Pérez y Grimalt-Santacana). 
En cambio, en la hiperhidrosis generalizada se caracteriza por la sudoración a lo largo de todo el cuerpo sin existir causa subyacente aparente, siendo las posibles etiologías las que la enmarcan en un trastorno de tipo neurológico, endocrino, infeccioso o farmacológico.

Los factores agravantes de la enfermedad están relacionados con el calor, el estrés, alteraciones en los órganos olfativo y gustativo y con una lesión neurológica denominada Síndrome de Frey que da lugar a hiperhidrosis local (Tabla 1) 14,15,16.

\section{CLÍNICA}

Los pacientes que suelen consultar son, normalmente, jóvenes, y en su mayoría mujeres, aunque los médicos y los pacientes a menudo no reconocen el hecho de que la hiperhidrosis primaria es una entidad relativamente frecuente y una afección que tiene tratamiento ${ }^{17,18}$. La historia clínica completa y un examen físico pormenorizado de los órganos y los sistemas contribuyen a descartar la existencia de la hiperhidrosis secundaria y posibles contraindica- ciones para algunos tipos de tratamiento, como debería tenerse en cuenta en el caso de un posible embarazo. Los síntomas asociados como la fiebre, los sudores nocturnos, la pérdida de peso, las linfadenopatías, la cefalea o palpitaciones deben alertar al clínico como base para investigar más a fondo la existencia de posibles causas secundarias, así como el impacto de la hiperhidrosis en la calidad de vida también debe ser evaluado por medio de la alguna escala $^{17}$.

La sintomatología habitual que puede aparecer en las personas que padecen hiperhidrosis está relacionada con un cuadro de hipersudoración, localizada en las palmas de las manos, zona axilar, craneofacial y/o podal, que se exacerba ante situaciones de estrés y que les produce una limitación en su vida social, laboral, académica... Los pacientes con hiperhidrosis palmar, al saludar intentan no dar la mano y en los pies evitan descalzarse ${ }^{18,19}$.

En la mayoría de los casos, se aprecia este aumento de sudor en la consulta que las personas relacionan con episodios en diferentes zonas corporales como la zona palmar, axilar, craneofacial y/o podal, que empeora en las si-

Tabla 1. Clasificación de las causas de hiperhidrosis secundaria (De Hornberger et al).

\begin{tabular}{|c|c|}
\hline Generalizada & $\begin{array}{l}\text { Medicamentos generalizados, abuso de sustancias, enferme- } \\
\text { dades cardiovasculares } \\
\text { insuficiencia respiratoria, infecciones, } \\
\text { tumores malignos, endocrino / metabólico } \\
\text { trastornos (tirotoxicosis, hipoglucemia, } \\
\text { feocromocitoma, acromegalia, tumor carcinoide), } \\
\text { neurológicas (Enfermedad de Parkinson) }\end{array}$ \\
\hline
\end{tabular}

Regional daño por apoplejía, nervio periférico, central

$\begin{array}{ll}\text { Regional } & \text { que causa anhidrosis localizada puede provocar } \\ & \text { sudoración compensatoria en otras áreas } \\ & \text { (lesión derrame cerebral, la médula espinal, } \\ & \text { neuropatía, síndrome de Ross) }\end{array}$

Focal

Focal síndrome de Frey, sudoración gustativa, ecrino nevus, trastorno de ansiedad social, hiperhidrosis focal unilateral

(por ejemplo, trastorno neurológico o tumor) 
tuaciones de estrés y que les produce una limitación en su vida social, laboral, académica... ${ }^{20}$

\section{DIAGNÓSTICO}

Existen diferentes métodos para realizar el diagnóstico, pero el más utilizado, barato y práctico es realizar una correcta historia clínica y una exploración física, siendo los criterios que se recomiendan para establecer el diagnóstico que la sudoración sea excesiva y visible con un intervalo de mas de seis meses de duración sin causa aparente con al menos dos de las siguientes características ${ }^{21}$ :

- Bilateral y simétrica relativamente.

- Menoscaba las actividades diarias.

- Frecuencia de al menos un episodio por semana.

- Edad de inicio inferior a 25 años.

- Historia familiar positiva.

- Cese de la sudoración focal durante el sueño.

Además, para evaluar la gravedad de la enfermedad existen diferentes formas, una de las más habituales es utilizar la Escala Severidad de la Hiperhidrosis (ESH) para medir la magnitud que ocasiona la enfermedad al paciente en las actividades diarias y el tipo de hiperhidrosis $^{22}$.
Para ello, los pacientes seleccionan la frase que mejor refleja su experiencia con la sudoración en el área afectada. Una puntuación de 3 o 4 indica la hiperhidrosis es severa, mientras que una puntuación de 1 o 2 indica que la hiperhidrosis es primaria, leve o moderada (véase Tabla 2) ${ }^{23}$.

Esta herramienta de diagnóstico rápido permite al clínico identificar el nivel de interferencia con las actividades diarias. Se trata de un instrumento de un solo punto que puede ser rápidamente administrado en forma escrita o entrevista, es fácil de entender, y no requiere ayudas para la realización. La validez y fiabilidad de la ESH se analizó mediante tres estudios que mostraron la fiabilidad y validez del mismo $^{24,25}$.

Otras técnicas complementarias para identificar zonas con hiperhidrosis son ${ }^{18,26,27}$ :

- Test del almidón y el yodo. Una vez bien seca la región a explorar, se pinta con una sustancia yodada, como povidona yodada, y se seca. Encima, se espolvorea almidón en polvo; inmediatamente, allí donde empieza a sudar se tiñe de color violeta oscuro.

- Violeta de genciana. Tras secar la región a explorar, se "pinta" con una gasa impregnada en violeta de genciana; el área afecta se tiñe.

- Pañuelo de papel. Colocar un pañuelo de papel sobre el área a explorar para verificar

Tabla 2. Escala de Severidad de la Hiperhidrosis.

Puntuación Grado de Severidad Tipo de Hiperhidrosis

I Mi transpiración nunca se notaba y nunca interfería con mis actividades diarias.

Hiperhidrosis Primaria, leve o moderada

Hiperhidrosis Primaria, leve o moderada

Hiperhidrosis Severa

III frecuentemente interfería con mis

IV
Mi transpiración era apenas tolerable y actividades diarias

Mi transpiración era tolerable, pero a veces interfería en mis actividades diarias

Mi transpiración era intolerable y siempre interfería con mis actividades diarias
Hiperhidrosis Severa 
dónde se moja es otra de las técnicas empleadas, más sencilla pero que no permite delimitar correctamente la zona.

- Medidas gravimétricas. Se puede medir la pérdida de agua transepidérmica en $\mathrm{g} / \mathrm{m} 2 /$ h2. Habitualmente sólo se utilizan en estudios experimentales.

\section{TRATAMIENTO}

Hay una amplia gama de modalidades disponibles para el tratamiento de la hiperhidrosis en el pie. Estos incluyen tratamientos conservadores (es decir, tratamientos tópicos y sistémicos) y quirúrgicos que varían su eficacia terapéutica dependiendo del grado de severidad, los efectos secundarios, el coste y la duración del efecto. En todos los pacientes que padecen hiperhidro- sis, es crucial medir la gravedad de la enfermedad por medio de la ESH para así determinar el adecuado curso del tratamiento y para poder monitorear los resultados del mismo ${ }^{28}$.

La evolución del tratamiento, así como el éxito y fracaso en el abordaje terapéutico en los pacientes con hiperhidrosis plantar estará relacionada con una mejora de una puntuación de la ESH de 4 o 3 a un 2 o 1 y el fracaso del tratamiento puede ser definido como ningún cambio en la puntuación de la ESH después de 1 mes de tratamiento o la falta de tolerabilidad del mismo

El algoritmo terapéutico del tratamiento para la hiperhidrosis plantar está relacionado como las líneas de actuación dependiendo de la puntuación en la ESH (véase Tabla 3).

El eje central de la primera línea de actuación se relaciona con la aplicación de manera tópica de cloruro de aluminio en los pies, que puede

Tabla 3. Algoritmo terapéutico del tratamiento de la hiperhidrosis plantar.

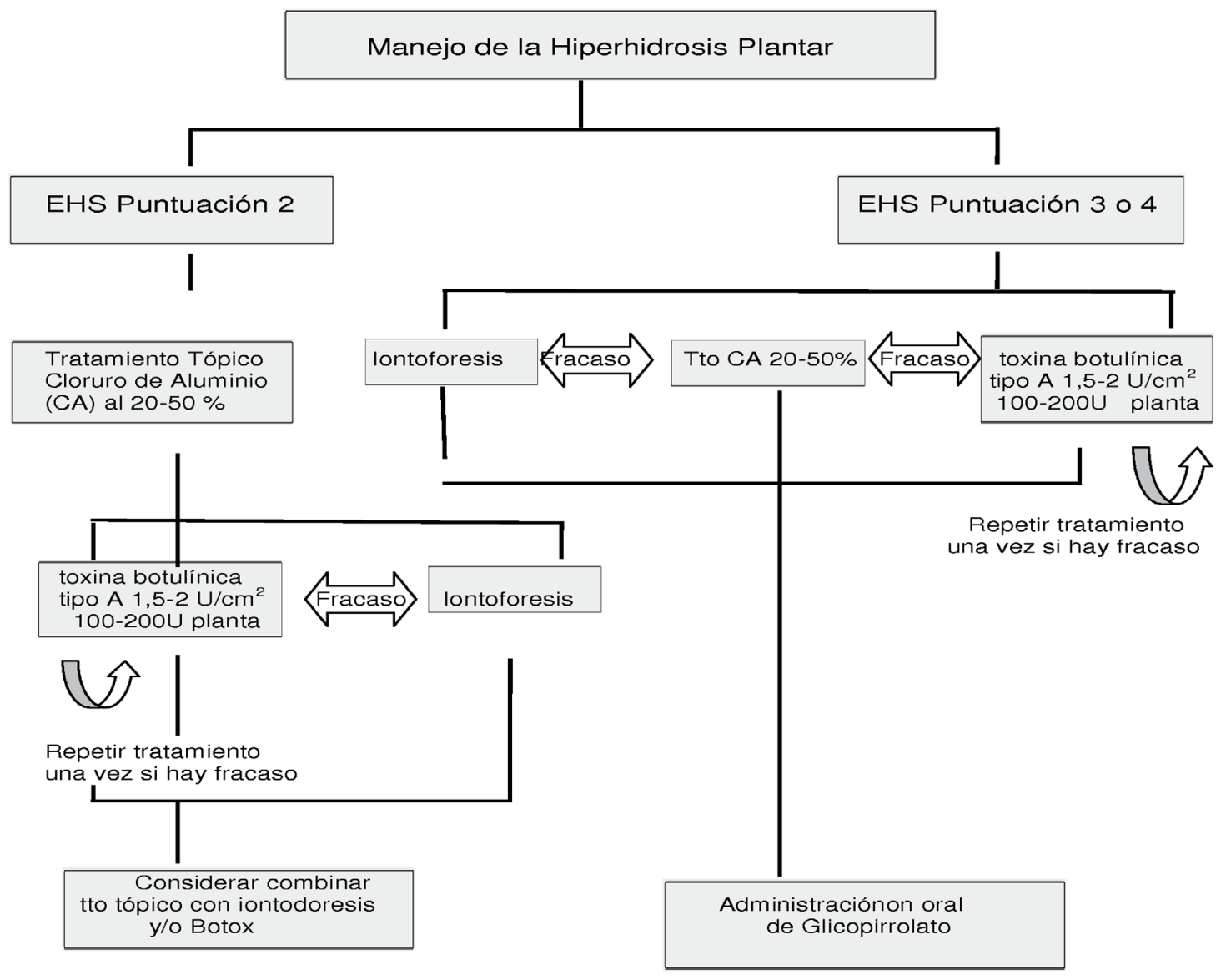


ser suficiente para la mayoría de los casos y que se puede combinar con otros tratamientos si el resultado es insuficiente a lo esperado.

A continuación, se recomienda la iontoforesis como el segundo paso en la terapia. Esta modalidad tendrá la eficacia más alta para la mayoría de los pacientes y tiene la ventaja de poder realizarse en casa, y de que el paciente realice el autotratamiento. Existen diferentes modelos de máquinas que galvanizan el agua. No se conoce bien el mecanismo por el cual el agua galvanizada produce, hasta en el $85 \%$ de los pacientes, una euhidrosis. Puede utilizarse, también, un anticolinérgico junto con el agua para que el tratamiento sea más efectivo. El único efecto secundario descrito es la sequedad excesiva, que puede producir fisuras en la piel. Su principal inconveniente es el coste del equipo, la educación inicial para realizar su uso y que al cesar el tratamiento, reaparece la hiperhidrosis inmediatamente ${ }^{18,29}$.

La tercera línea de abordaje terapéutico está relacionada con la administración de inyecciones subcutáneas de la toxina botulínica tipo A, que actúa bloqueando la liberación neuronal de la acetilcolina de la unión presináptica neuromuscular y en las neuronas autonómicas y que puede reducir temporalmente la producción de sudor. Por lo general dura de 3 a 4 meses como mínimo, sin embargo, puede durar hasta 7 meses, hasta que las fibras nerviosas sudomotoras han regenerado los receptores de acetilcoli$\mathrm{na}^{30,31}$.

El tratamiento consiste en múltiples inyecciones subcutáneas, por lo general son necesarias entre 20 y 40 en la zona afectada, que se habrá identificado previamente, diluyendo 50 UI en 4 cc de suero fisiológico, pudiéndose aplicar hasta 200 UI en un área previa anestesia local del pie, debido al malestar que genera su aplicación en la planta de los pies. Los efectos secundarios se asocian a debilidad transitoria de la zona. Se recomienda su uso cuando fallan otras opciones y cuando la gravedad es lo suficientemente notoria e interfiere con las actividades diarias de la persona ${ }^{32,33}$.

Para la cuarta línea de actuación se recomienda la administración oral de anticolinérgicos (glicopirrolato, atropina, inhibidores de la acetilcolina), con el que se consigue una disminución del sudor de forma generalizada, por lo que son poco utilizados en la hiperhidrosis localizada en los pies y se utilizan, básicamente, en la hiperhidrosis generalizada y en algunos casos de hiperhidrosis en los que falla el resto de los tratamientos Suelen ser mal tolerados por sus efectos secundarios: xerostomía, midriasis, cicloplejía, disfunción intestinal y vesical (hasta puede producir íleo paralítico y retención urinaria)... ${ }^{34,35}$

\section{CONCLUSIONES}

La hiperhidrosis en el pie es un trastorno frecuente con importantes consecuencias clínicas, siendo la presentación clínica variable, así como los patrones de distribución, la severidad e impacto en las personas.

La variedad de opciones de tratamiento disponibles se deben enfocar en función de la gravedad y pueden ser medidas por medio de la ESH que facilita en la mayoría de los casos, la adaptación del tratamiento a cada paciente en la búsqueda de un resultado satisfactorio.

Sin embargo, además de la puntuación de la ESH y la localización, se deben tener en cuenta otros factores como la duración del efecto, los efectos secundarios, las complicaciones, el coste económico, así como la adherencia terapéutica del paciente, relacionada con la toma de decisiones en el abordaje terapéutico.

\section{BIBLIOGRAFÍA}

1. Sato K, Kang WH, Saga K, Sato KT. Biology of sweat glands and their disorders. II. Disorders of sweat gland function. J Am Acad Dermatol 1989; 1: 713-726.

2. Worle B, Rapprich S, Heckmann M. Definition and treatment of primary hyperhidrosis. J Dtsch Dermatol Ges 2007; 5: 625-628.

3. Callejas-Pérez MA, Grimalt-Santacana R. Hiperhidrosis primaria: un reto terapéutico actual. JANO 2006; 1591: 37-39. 
4. Ramos R, Moya J, Pérez J, Villalonga R, Morera R, Pujol R, Ferrer G. Hiperhidrosis primaria: Estudio prospectivo de 338 pacientes. Med Clin (Barc) 2003;121:201-3.

5. Walling HW. Primary hyperhidrosis increases the risk of cutaneous infection: a case-control study of 387 patients. J Am Acad Dermatol 2009; 61: 242-246.

6. Leung AK, Chan PY, Choi MC. Hyperhidrosis. Int J Dermatol 1999;38:561-7.

7. Solish N, Wang R, Murria CA. Evaluating the patient presenting with hyperhidrosis. Torac Surg Clin. 2008; 18:133-40.

8. Walling HW, Swick BL.Treatment Options for Hyperhidrosis. Am J Clin Dermatol 2011; 12 (5): 1-11.

9. Cerfolio RJ, De Campos JR, Bryant AS, Connery CP, Millar DL, DeCamp MM, et al. The Society of Thoracic Surgeons Expert Consensus for the Surgical Treatment of Hyperhidrosis. Ann Thorac Surg 2011;91:1642-8.

10. Rzany BBR, Spinner D. Interventions for localised excessive sweating (Protocol). Cochrane Database of Systematic Reviews 2000, Issue 3.

11. Grimalt R, Callejas MA, editores. Hiperhidrosis. Diagnóstico actual y tratamiento. 1. ${ }^{\mathrm{a}}$ ed. Madrid: Médica Panamericana; 2004.

12. Kao MC, Lee WY, Yip KM, Hsiao YY, Lee YS, Tsai JC. Palmar hyperhidrosis in children: treatment with video endoscopy laser sympathectomy. J Ped Surg 1994; 29: 387-91.

13. Ro KM, Cantor RM, Lange KL, Ahn SS. Palmar hyperhidrosis: evidence of genetic transmission. J Vasc Surg 2002;35:382-6.

14. Álvarez CC, Rodríguez E, García AM, Galache C, Blanco S, et al. Síndrome auriculotemporal de Frey. Un caso de presentación bilateral en un lactante. Med Cutan Iber Lat Am 2007;35(6):295-297.

15. Altman R, Schwartz R. Emotionally induced hyperhidrosis. Cutis 2002;69:336-8.

16. Bergmann I, Duauphin M, Naumann M, Flachenecker P, Mullges W, Koltzenburg M, et al. Selective degeneration of sudomotor fibers in Ross syndrome and successful treatment of compensatory hyperhidrosis with botulinum toxin. Muscle Nerve 1998;21:1790-3.

17. Callejas M A, Grimalt R, Cladellas E. Hyperhidrosis Update. Actas Dermosifiliogr. 2010; 101(2):110118

18. Rubio M M, Martínez S, Mármol E E, Baldó X, Penagos JC, Sebastián F. Aproximación diagnóstica y terapéutica a la hiperhidrosis. JANO 2010, 51-55.

19. Wolosker N, Yazbek G, Milanez de Campos JR, Kauffman P, Ishy A, Puech- Leao P. Evaluation of plantar hyperhidrosis in patients undergoing videoassisted thoracoscopic sympathectomy. Clin Auton Res 2007;17(June (3)):172-6.

20. Ramos R, Moya J, Turo'n V, Pe'rez J, Villalonga R, Morera R, Perna V, Ferrer G. Primary hyperhidrosis and anxiety: a prospective preoperative survey of 158 patients. Arch Bronconeumol 2005;1 (2):88-92.

21. Hornberger J, Grimes K, Naumann M, Glaser DA, Lowe NJ, Naver H, Ahn S, Stolman LP.Recognition, diagnosis, and treatment of primary focal hyperhidrosis. J Am Acad Dermatol 2004, 51, (2): 274:286.

22. Haider A, Solish N. Focal hyperhidrosis: diagnosis and management. CMAJ 2005;172:69-75.

23. Solish N, Benohanian A, Kowalski JW. Prospective open-label study of botulinum toxin type A in patients with axillary hyperhidrosis. effects on functional impairment and quality of life. Dermatol Surg 2005;31:405-13.

24. Solish N, Benohanian A, Kowalski JW. Prospective open-label study of botulinum toxin type A in patients with axillary hyperhidrosis: effects on functional impairment and quality of life. Dermatol Surg 2005;31:405-13.

25. Kowalski JW, Eadie N, Lai PY, et al. Validity and reliability of the Hyperhidrosis Disease Severity Scale (HDSS). Poster Presented at: 62nd Annual Meeting of the American Academy of Dermatology; February 6-10, 2004; Washington, DC. Poster P198. J Am Acad Dermatol 2004; 50:51.2006.

26. Swinehart J.M. Treatment of axillary hyperhidrosis: combination of the starch-iodine test with the tumescent liposuction technique. Dermatol Surg. 2000; 26:392-6.

27. Hund M, Kinkelin I, Naumann M, Hamm H. Definition of axillary hyperhidrosis by gravimetric assegssment. Arch Dermatol. 2002; 138:539-41.

28. Solish N, Bertucci V, Dansereau A et al. A comprehensive approach to the recognition, diagnosis, and severity-based treatment of focal hyperhidrosis: recommendations of the Canadian Hyperhidrosis Advisory Committee. Dermatol Surg 2007; 33: 908-923.

29. Stolman LP. Hyperhidrosis. Medical and Surgical Treatment. Eplasty. 2008; 8: e22.

30. Heckmann M, Ceballos-Baumann AO, Plewig G. Botulinum toxin A for axillary hyperhidrosis (excessive sweating). N Engl J Med 2001;344:488 -93. 
31. Naumann M, Lowe NJ. Botulinum toxin type A in treatment of bilateral primary axillary hyperhidrosis: randomised, parallel group, double blind, placebo controlled trial. BMJ 2001;323:596-9.

32. Glogau RG. Botulinum A neurotoxin for axillary hyperhidrosis. No sweat Botox. Dermatol Surg 1998;24:817-9.

33. Hsu C, Shia SE, Hsia JY, Chuang CY, Chen CY. Experiences in thoracoscopic sympathectomy for axillary hyperhidrosis and osmidrosis. Arch Surg 2001;136:1115-7.

34. Reisfeld R, Berliner KI. Evidence-based review of the nonsurgical management of hyperhidrosis. Thorac Surg Clin. 2008; 18:157-66.

35. Mack GW, Shannon LM, Nadel ER. Influence of betaadrenergic blockade on the control of sweating in humans. Appl Physiol 1986; 61:1701-5. 